\title{
LONGITUDINAL VARIATIONS, THE OPPOSITION EFFECT AND MONOCHROMATIC ALBEDOS FOR MARS*
}

\author{
WILLIAM M. IRVINE and JAMES C. HIGDON \\ Dept. of Physics and Astronomy, University of Massachusetts, Amherst, Mass., U.S.A. \\ and \\ SUSAN J. EHRLICH \\ Smith College, Northhampton, Mass., U.S.A.
}

\begin{abstract}
Observations of Mars previously reported in 10 narrow bands between $3150 \AA$ and $1.06 \mu$ and in UBV are analyzed for brightness variations which correlate with longitude of the central meridian. Such an effect is found for $\lambda \geqslant 5000 \AA$, with some evidence for such a correlation at $\lambda=4570 \AA$. The data are then corrected to the mean (over longitude) brightness and a linear phase curve fitted to those observations with phase angle $i \geqslant 15^{\circ}$. An opposition effect (anomalous brightening at small phase angles) is found for wavelengths $\lambda \leqslant 5500 \AA$, in contrast to a result previously reported. The magnitude at zero phase, phase coefficient, and monochromatic albedo are computed for Mars as a function of wavelength.
\end{abstract}

\section{Introduction}

Multicolor photoelectric photometry of Mars between 1963 and 1965 has been reported in two previous papers (Irvine et al., 1968a, Paper I; Irvine et al., 1968b, Paper II). The observations were made using 10 narrow bands isolated by interference filters between $3150 \AA$ and $1.06 \mu$ and also in UBV. The narrow bands were labeled $v-u-s-p-m-l-k-h-g-e$ as shown in Table I of Hopkins and Irvine (1969). The observations were conducted from two sites, one in South Africa and one in France; for the present paper these results are combined.

\section{Longitudinal Variations}

From the data presented in Paper I and Paper II we selected those observations which were not denoted by an asterisk; that is, we selected observations made under superior observing conditions. We then further selected those observations corresponding to phase angles $i \geqslant 15^{\circ}$. A linear least squares fit to this data was made, and the residuals $R$ were plotted versus longitude of the central meridian on Mars $\omega$. The correlations found are shown in Figure 1. The longitudinal effect is easily observed. Its magnitude increases with wavelength out to the long wavelength limit of our observations $(1.06 \mu)$. It is clearly visible for wavelengths as short as $5000 \AA$, and there is some evidence for the effect in the band at $4570 \AA$. The solid line in Figure 1 is a least squares fit using a 6th-order polynomial, with the obvious constraint that the curve and its first derivative be periodic with period $2 \pi$. Note that the planetocentric declination of the Earth was $D_{E} \simeq 20^{\circ}$ during the periods of observation.

\footnotetext{
* Contribution from the Four College Observatories, \#64.
} 

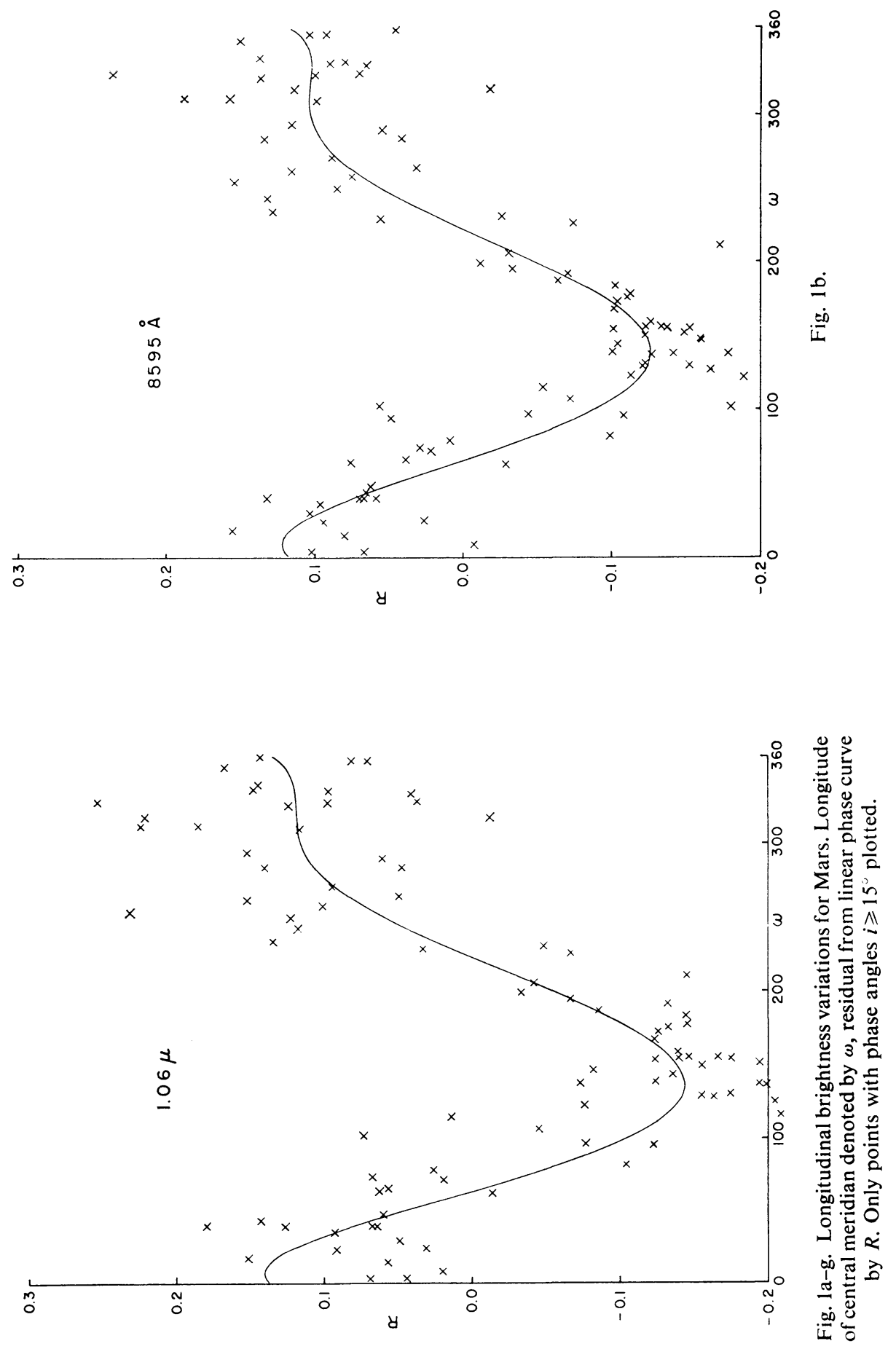

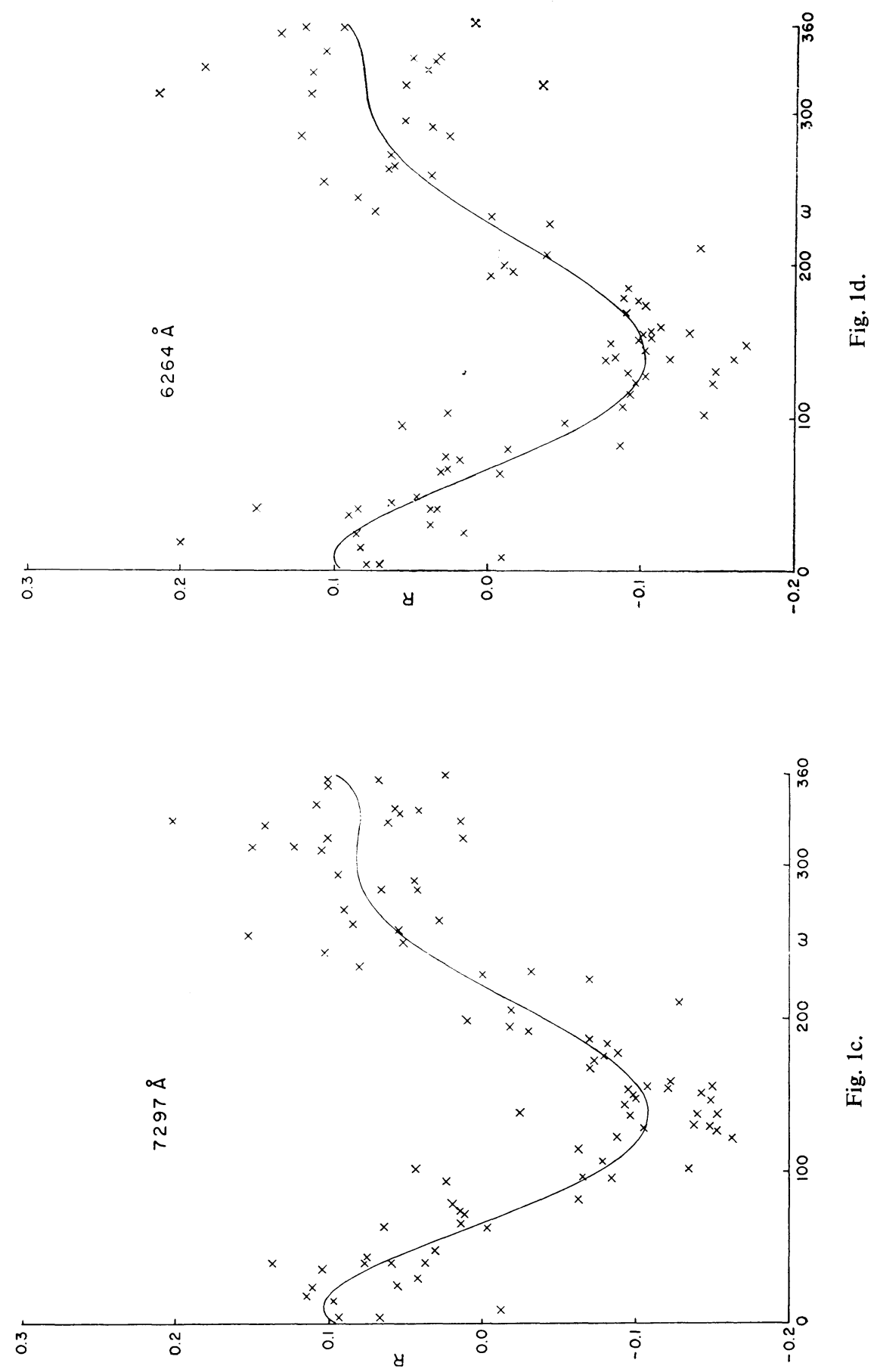

11 - P.A. 

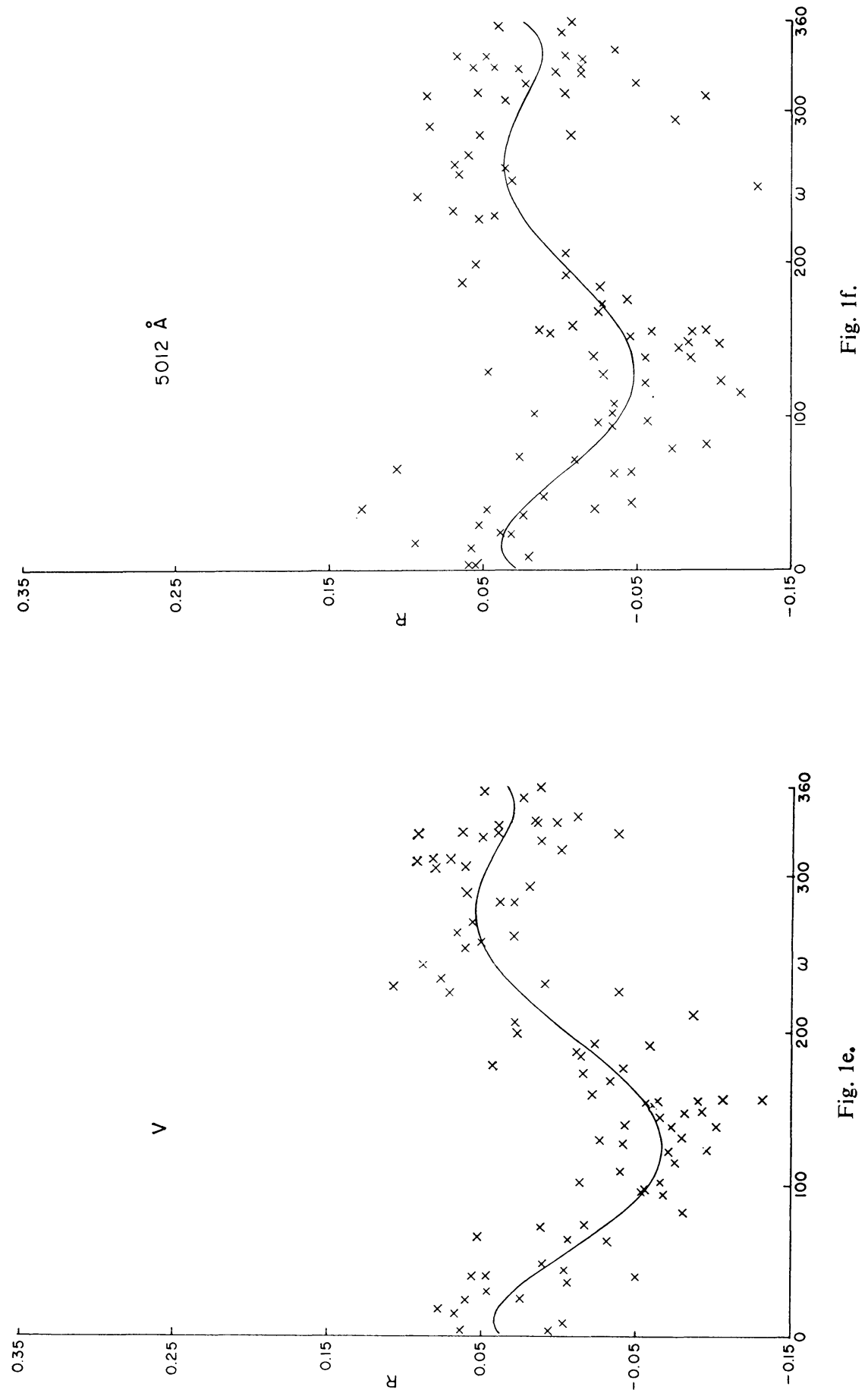


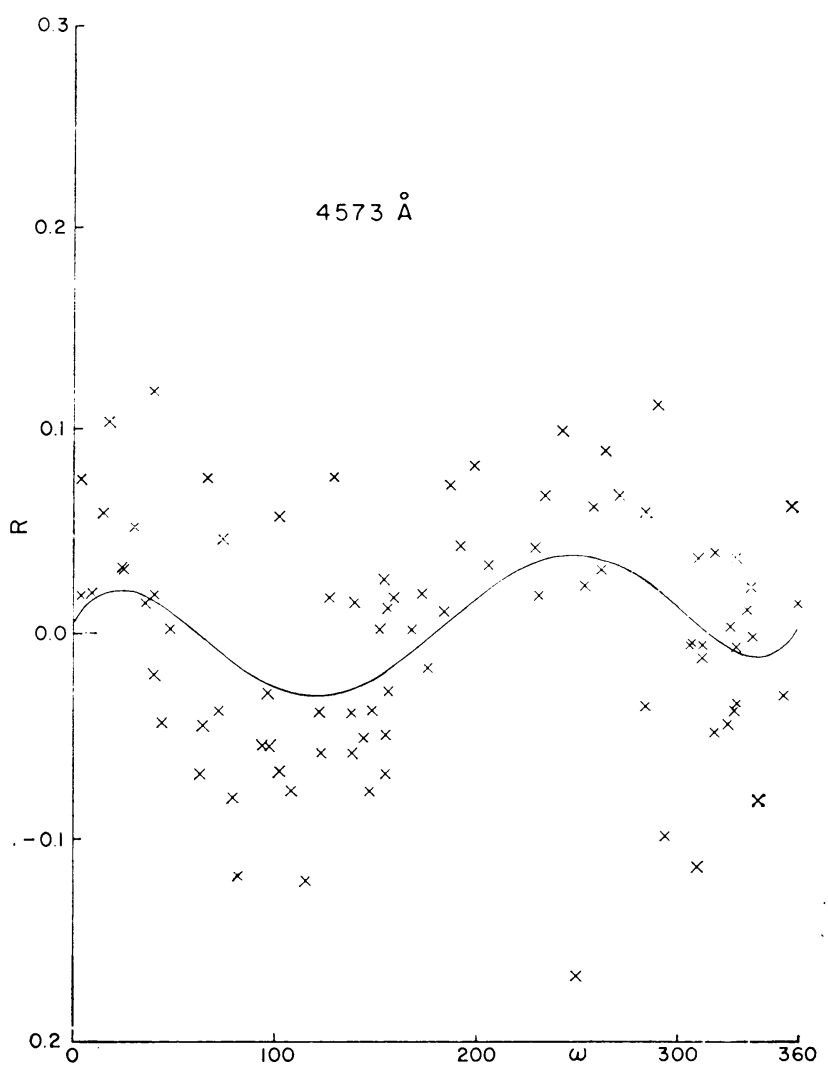

Fig. 1g.

For the shorter wavelengths the correlation observed no longer seems related to surface features and is apparently not statistically significant (see for example Figure 2). This is to be expected from the well known loss of observable surface detail on Mars at wavelengths $\lambda \lesssim 4550 \AA$.

\section{Phase Curves and the Opposition Effect}

The observations in filters $m-l-k-h-g-e$ and $V(\lambda \geqslant 4570 \AA)$ were then corrected to a mean longitudinal brightness using the least squares fit illustrated in Figure 1. The resulting data, and also the corresponding observations for wavelengths $\lambda \leqslant 4500 \AA$, were then plotted versus phase angle and a linear least squares fit was made (remember that this data includes only observations for $i \geqslant 15^{\circ}$ ). The resulting straight line is the full line shown in Figure 3, and the corresponding magnitudes at zero phase $m(1,0)$ (all the data have been reduced to unit distance) and phase coefficient $a$ are given in Table I, columns 2 and 3 . We note that the narrow band color of the sun is zero on our magnitude system. Standard errors for $m(1,0)$ are typically $\sim 0.015 \mathrm{~m}$. The observational data for phase angles $i \leqslant 15^{\circ}$ were then corrected for the longitudinal effect and 

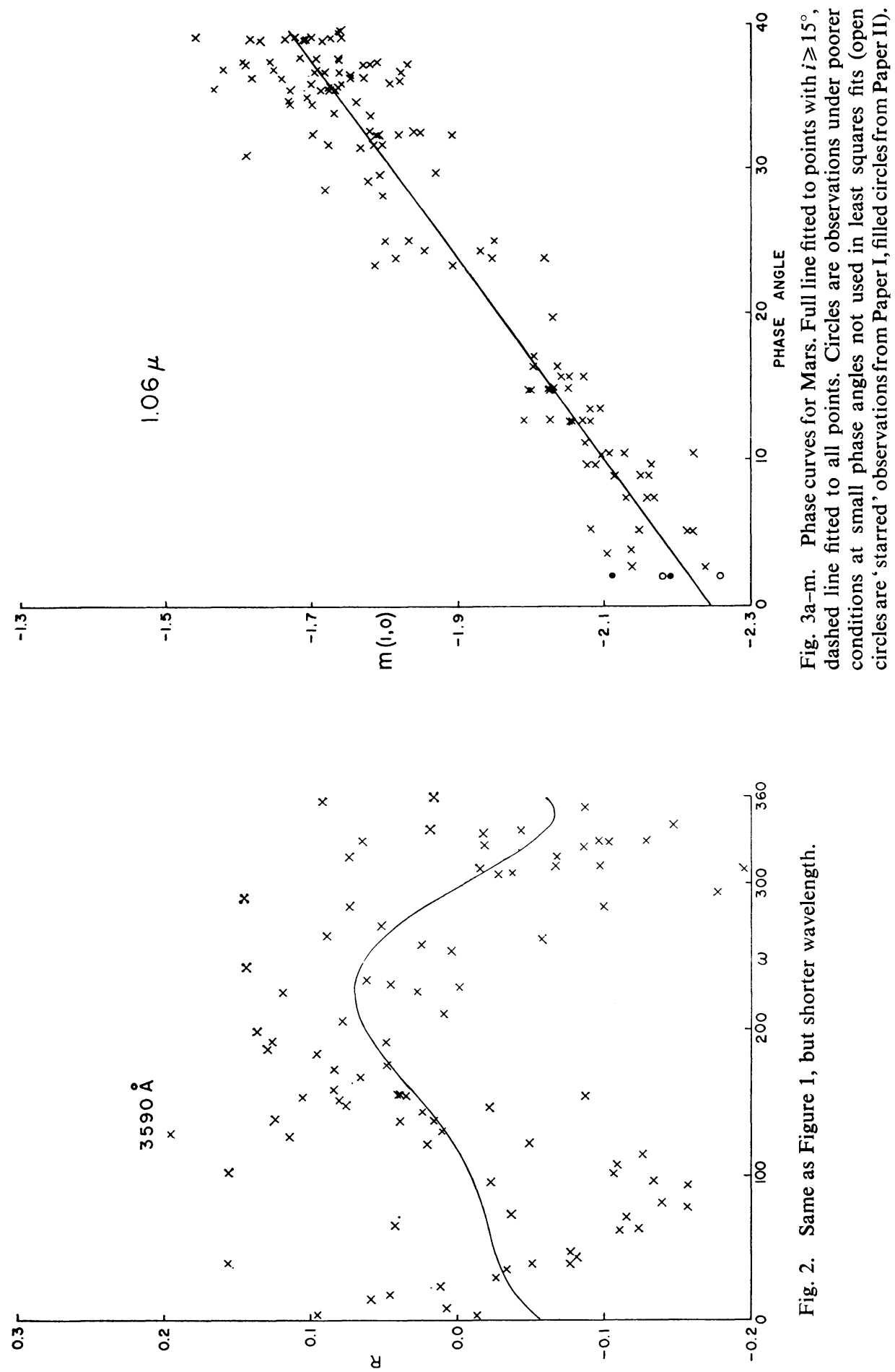

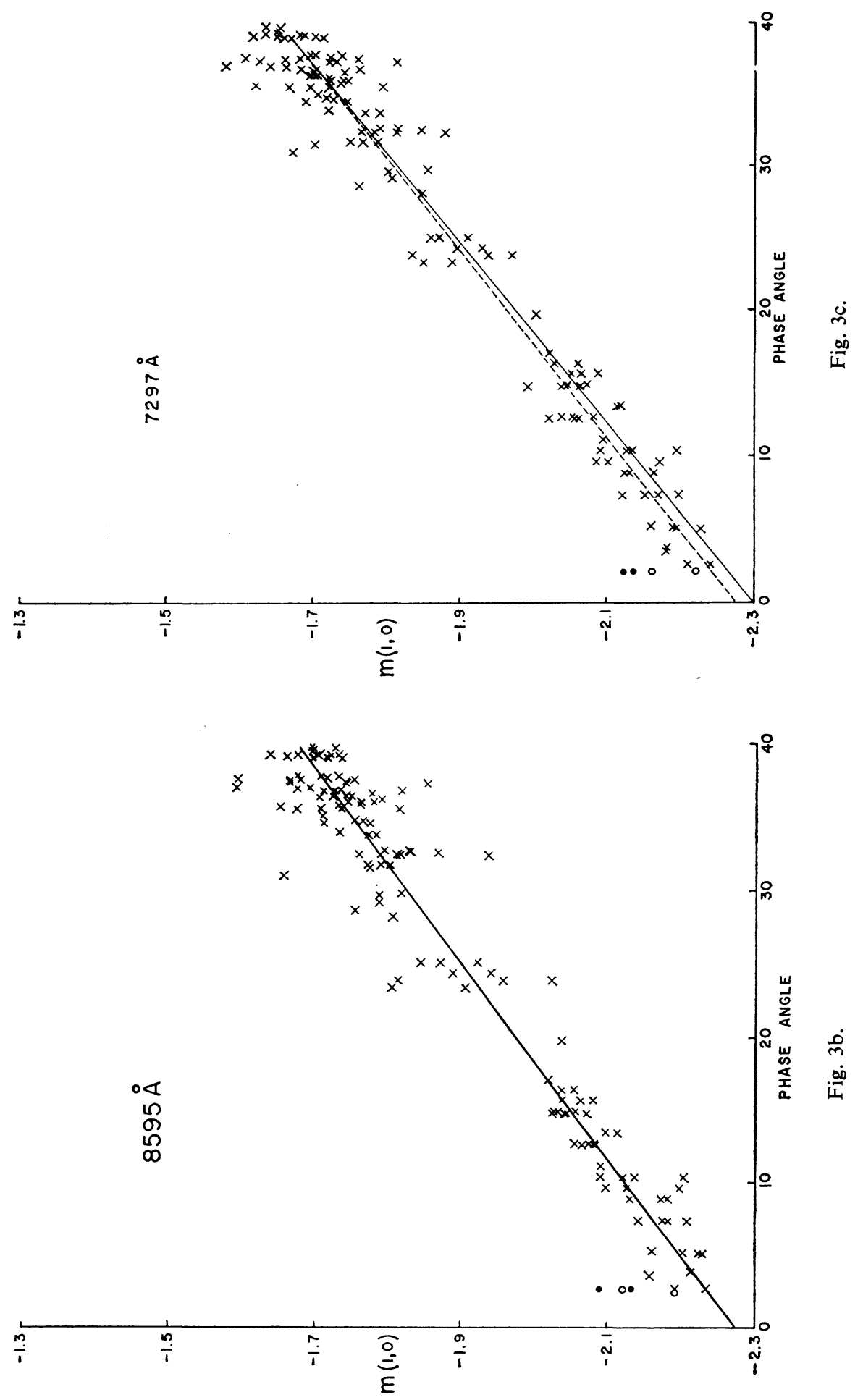

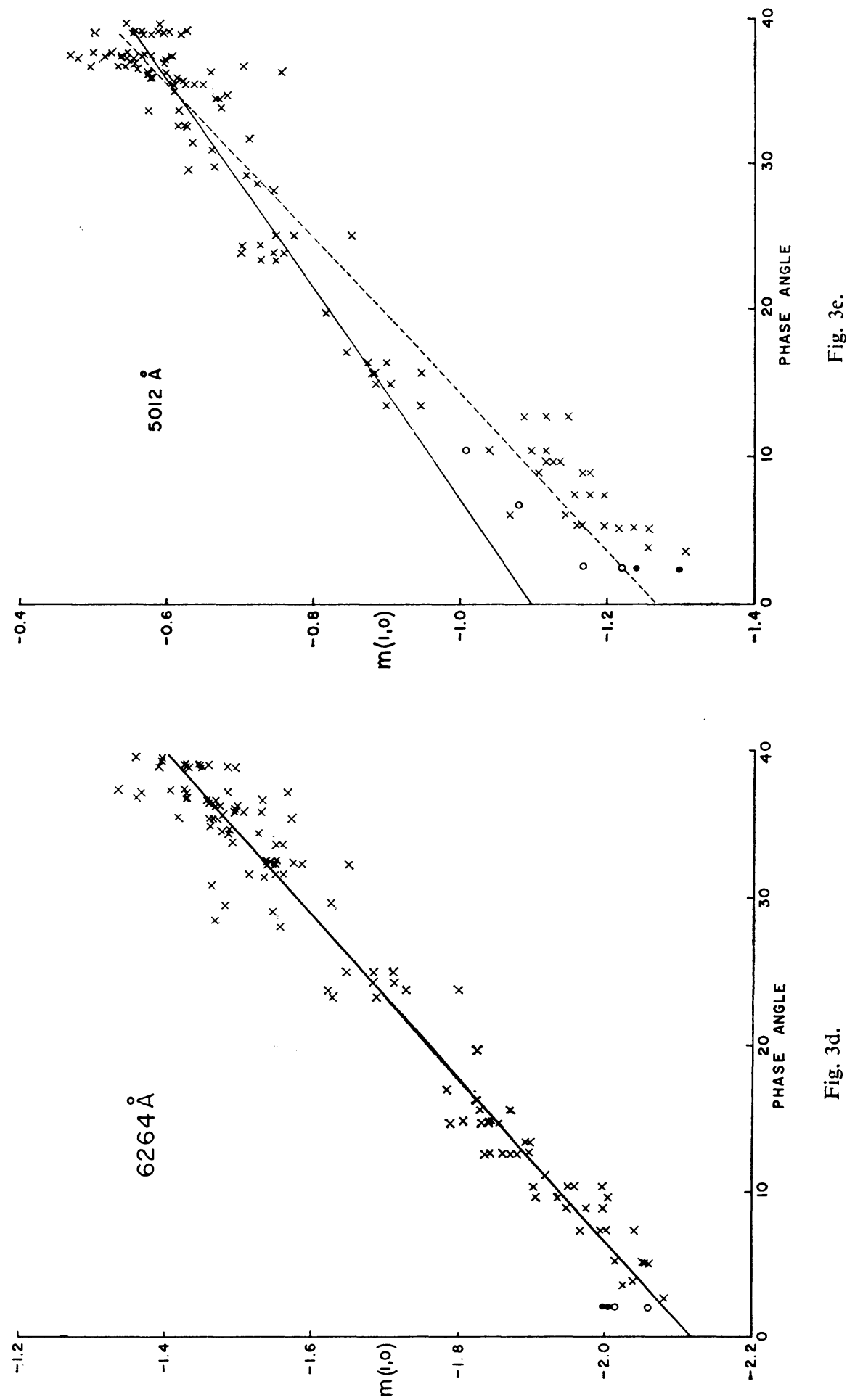

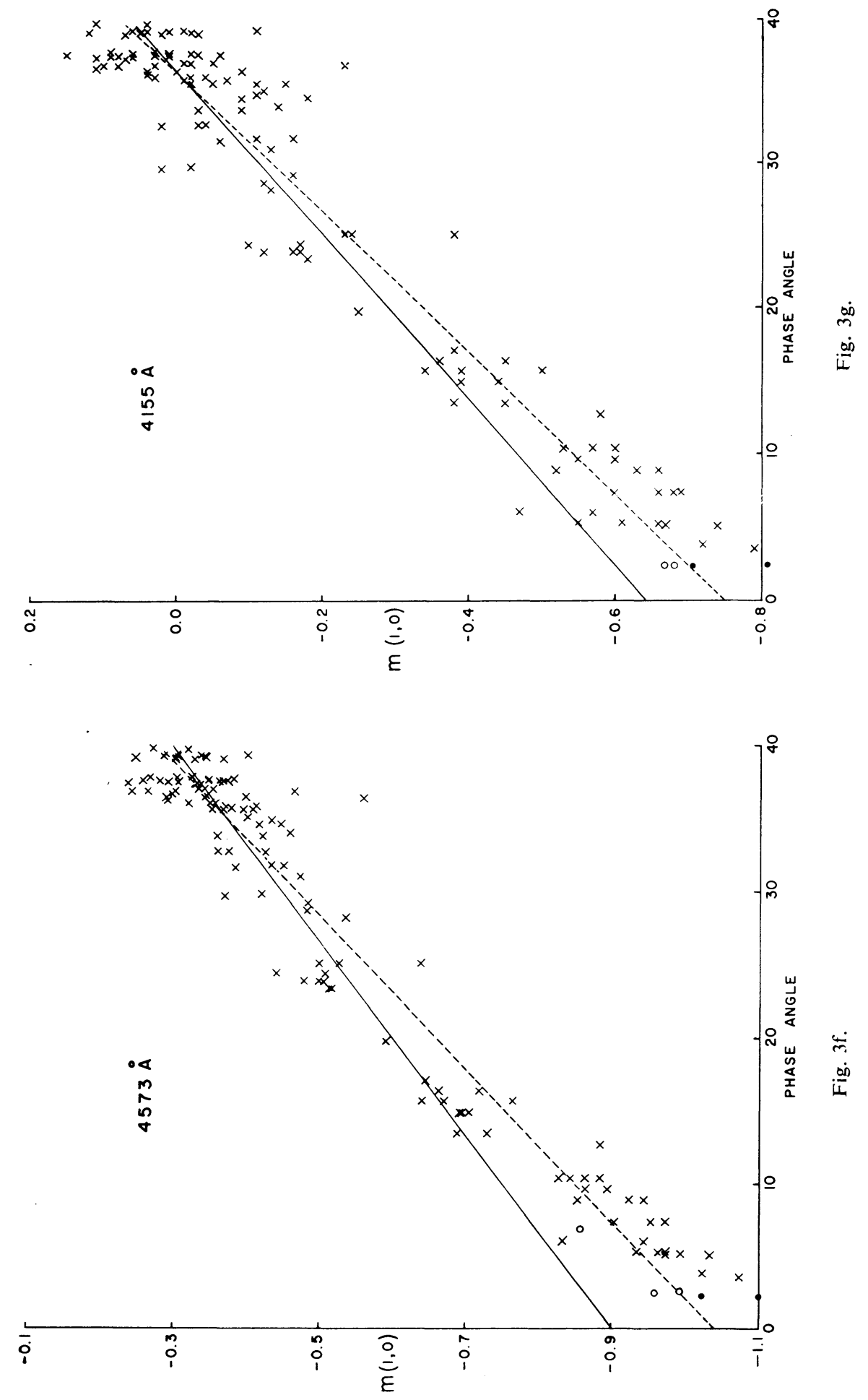

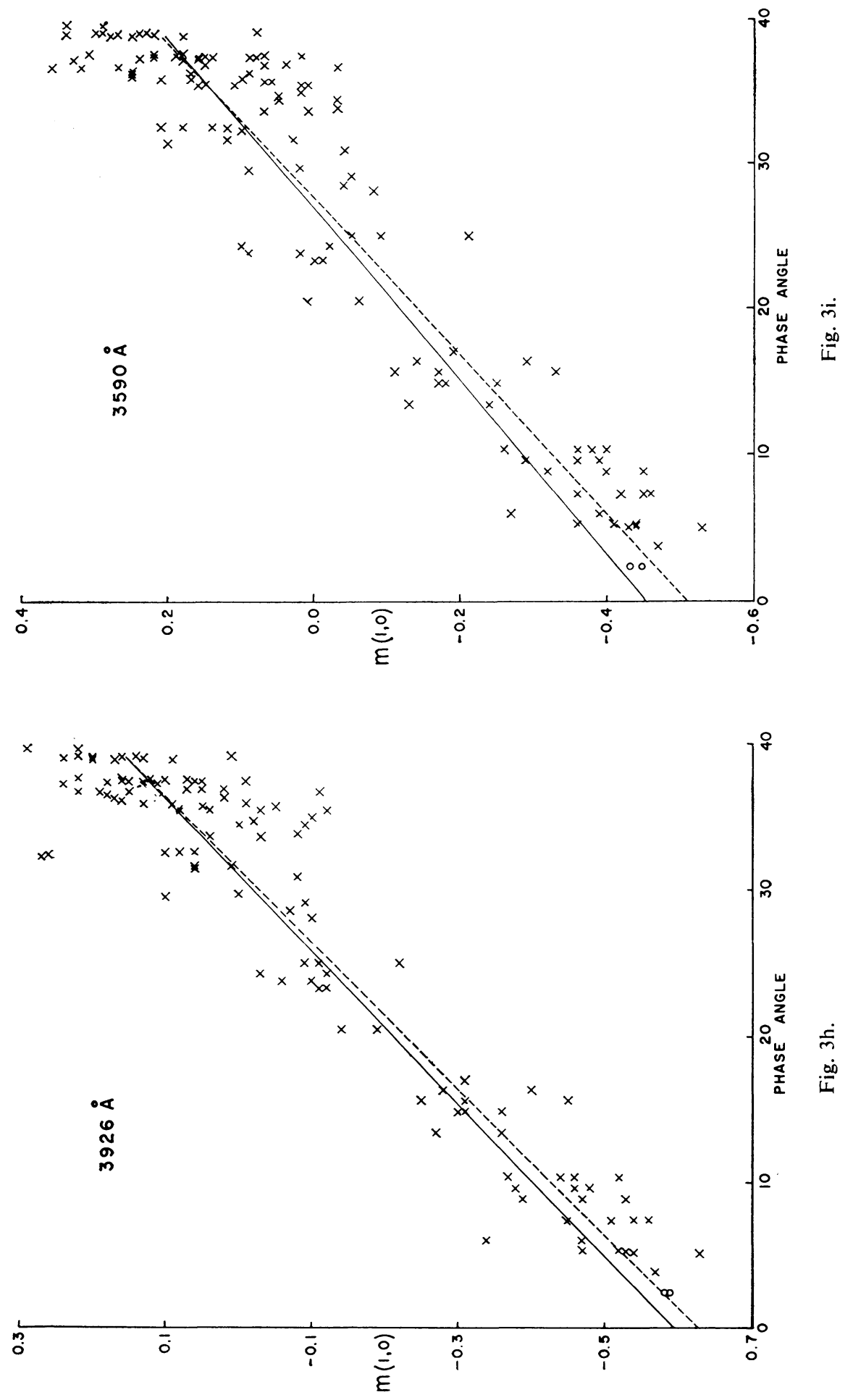

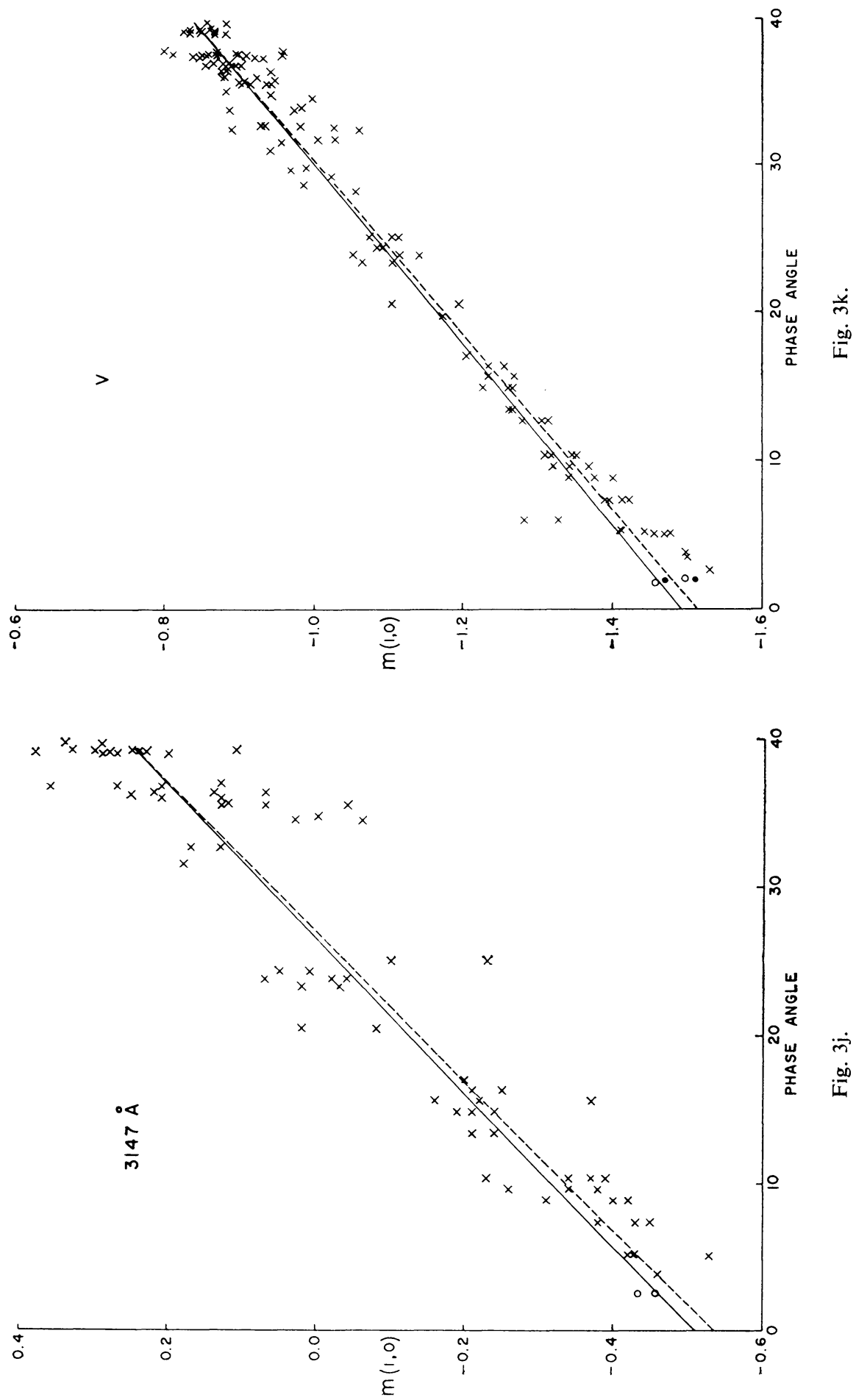

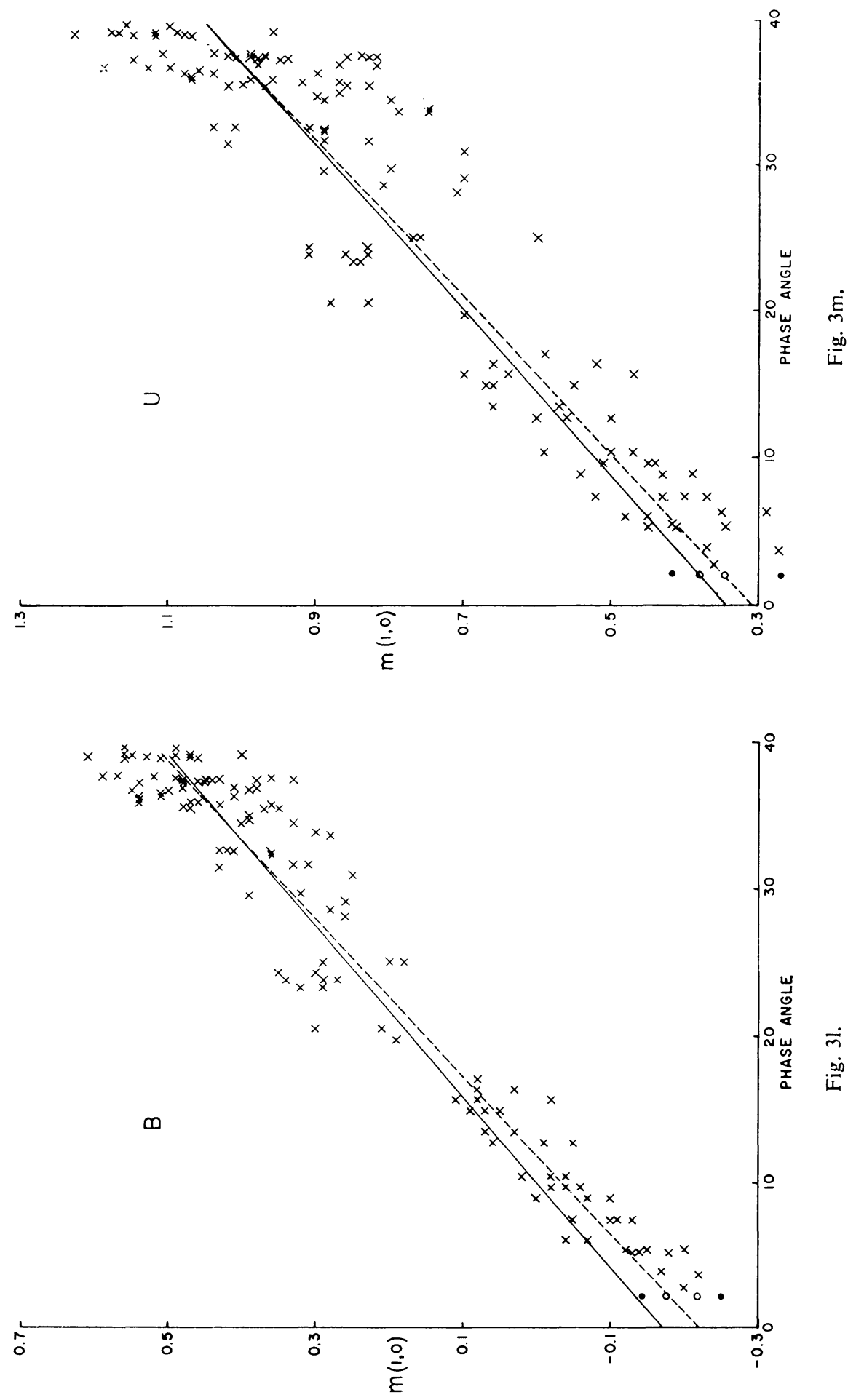
TABLE I

Spectral reflectivity of Mars

\begin{tabular}{llllllll}
\hline$\lambda$ & $m(1,0)^{\mathrm{a}}$ & $a^{\mathrm{a}}$ & $m(1,0)^{\mathrm{b}}$ & $a^{\mathrm{b}}$ & \multicolumn{1}{l}{$m(1,0)^{\mathrm{c}}$} & \multicolumn{1}{c}{$\Delta M_{0}^{\mathrm{d}}$} & \multicolumn{1}{l}{$A$} \\
\hline $3147 \AA$ & -0.51 & 0.019 & -0.54 & 0.020 & $-0.60 \pm 0.05$ & $0.09 \pm 0.05$ & 0.052 \\
3590 & -0.45 & 0.017 & -0.51 & 0.019 & $-0.58 \pm 0.05$ & $0.13 \pm 0.05$ & 0.053 \\
3926 & -0.60 & 0.019 & -0.63 & 0.020 & $-0.70 \pm 0.04$ & $0.10 \pm 0.04$ & 0.057 \\
4155 & -0.65 & 0.018 & -0.75 & 0.021 & $-0.81 \pm 0.06$ & $0.16 \pm 0.06$ & 0.006 \\
4573 & -0.90 & 0.015 & -1.04 & 0.019 & $-1.13 \pm 0.08$ & $0.23 \pm 0.10$ & 0.086 \\
5012 & -1.10 & 0.014 & -1.27 & 0.018 & $-1.38 \pm 0.09$ & $0.28 \pm 0.15$ & 0.112 \\
6264 & -2.12 & 0.018 & -2.12 & 0.018 & $-2.12 \pm 0.01$ & $0 \pm 0.02$ & 0.244 \\
7297 & -2.30 & 0.016 & -2.27 & 0.016 & $-2.27 \pm 0.02$ & $0 \pm 0.02$ & 0.308 \\
8595 & -2.27 & 0.015 & -2.27 & 0.015 & $-2.27 \pm 0.01$ & $0 \pm 0.02$ & 0.322 \\
$1.06 \mu$ & -2.25 & 0.015 & -2.24 & 0.015 & $-2.24 \pm 0.01$ & $0 \pm 0.02$ & 0.314 \\
$U$ & 0.34 & 0.018 & 0.31 & 0.019 & $0.22 \pm 0.07$ & $0.12 \pm 0.07$ & 0.052 \\
$B$ & -0.17 & 0.017 & -0.22 & 0.019 & $-0.32 \pm 0.04$ & $0.15 \pm 0.04$ & 0.074 \\
$V$ & -1.49 & 0.016 & -1.52 & 0.016 & $-1.58 \pm 0.04$ & $0.09 \pm 0.04$ & 0.154 \\
& & & & & & & \\
\hline
\end{tabular}

a Linear fit to data with $i \geqslant 15^{\circ}$.

${ }^{b}$ Linear fit to data at all $i$.

- Including estimated opposition effect.

d Column 2 minus column 6.

added to the plots, the least squares fit performed for all the data (dashed line in Figure 3 ), and the resultant intercept and slope listed in columns 4 and 5 of Table I. No significant change in the mean curve was found for filters $k-h-g$ - or $e(\lambda \geqslant 6250 \AA)$. An opposition effect (anomalous brightening for small phase angles) was, however, observed for filter $V$ and shorter wavelengths. This finding is in contradiction to the result previously reported (Irvine et al., 1968b) for this data, although anomalous brightening at the oppositions of 1967 and 1969 has been reported by Bugaenko et al. (1967), O'Leary (1967), and Murphy (1969). A (necessarily rough) extrapolation of our results to zero phase results in the values of $m(1,0)$ shown in column 6 of Table I, where the errors listed are 'eyeball' estimates. We also list the difference $\Delta M_{0}$ between the $m(1,0)$ in columns 2 and 6 of Table I (i.e., the 'magnitude' of the opposition effect).

A comment on the internal consistency of our results is in order here. The phase coefficients $a$ listed in column 3 of Table I appear anomalously small at $\lambda 4573$ and $\lambda 5012$ and rather large at $\lambda 6264$, both compared to the other narrow band data and also to the broad band ( $B$ and $V$ ) results. The wavelength range $5000 \leqslant \lambda \leqslant 4500 \AA$ will, of course, be most subject to changes in the 'blue haze', and our curves may be weighted by unusual atmospheric conditions. We also note from Paper II that the observations near opposition in bands $\lambda 4155, \lambda 4573$, and $\lambda 5012$ may be anomalously bright because of uncertainties in transformation to the standard magnitude system. The combination of these effects makes the value of $\Delta M_{0}$ in column 9 of Table I particularly uncertain for $\lambda 4573$ and $\lambda 5012$.

Our results for the opposition effect may be compared with those of O'Leary (1967) and O'Leary and Rea (1968). We do not confirm the existence of an opposition effect 
at wavelengths $\lambda>6000 \AA$, as those authors report. Rather it seems that at least part of the apparent effect in their data may be due to the selection of an asymptotic phase coefficient $a$ (for $i>16^{\circ}$, derived ultimately from Wooley et al. (1955)) which is too small, and, in the case of band $R(0.7 \mu)$, their choice of an $m(1,0)$ from the linear extrapolation (column 2 of Table I) which is fainter by about $0.06 \mathrm{~m}$ than is indicated by our data.

At wavelengths $\lambda \leqslant 5500 \AA$ the opposition effect which we observe is significantly less than that reported by O'Leary and Rea, and does not show the strong wavelength dependence which they report. In fact our results could be read as indicating no wavelength dependence of the effect for $5500 \AA \geqslant \lambda \geqslant 3150 \AA$. At $U$ this difference is in part the result of our finding a 'no-opposition-effect' $m(1,0)$ of 0.34 , considerably brighter than used by O'Leary and Rea and derived from de Vaucouleurs (1964). For $B$ and $V$ the difference may be partly due to O'Leary's observations extending to smaller phase angles, and conservatism on our part in the extrapolation of our results.

On the other hand, our observations were made during a different opposition, and parameters such as atmospheric aerosol content may play an important role in determining both $a$ and $m$. This discussion points out the difficulty of determining the magnitude of the opposition effect on a planet like Mars, for which atmospheric and surface conditions change both during an apparition and from apparition to apparition.

\section{Albedos}

Values of the geometric albedo including the opposition effect could be obtained from the values of $m(1,0)$ in column 6 of Table I using the standard formula (e.g., Paper II). The relatively large uncertainties in $\Delta M_{0}$ make this appear unprofitable, however. Rather we shall use the values in columns 4 and 5 to determine $p$, and Russell's Rule (Paper II) to find the phase integral $q$ and the spherical albedo $A=p q$; note that the first-order inclusion of the opposition effect increases $p$ and decreases $q$ by the same factor, so that $A$ is left unchanged. Values of $A$ calculated in the manner described, using parameters given in Paper II for the semi-diameter of Mars and the magnitude of the Sun $(V=-26.81)$, are listed in the last column of Table I. They fall, not surprisingly, between the values previously quoted in Papers I and II.

\section{Acknowledgments}

The observations of Mars discussed here were obtained under a grant to Professor Donald H. Menzel at the Harvard College Observatory. The present research was supported in part by NASA grant NGR 22-010-023 and NSF grant GP 7793 to the University of Massachusetts. Computations were performed principally at the Amherst College Computing Center, and the assistance of their staff is gratefully acknowledged. We are also grateful to Mr. Neil Hopkins for his aid. 


\section{References}

Bugaenko, L. A., Koval', I. K., and Morozhenko, A. V.: 1967, Trans. IAU (Commission 16), Prague.

de Vaucouleurs, G.: 1964, Icarus 3, 187.

Hopkins, N. B. and Irvine, W. M.: 1969, in this volume, p. 349.

Irvine, W. M., Simon, T., Menzel, D. H., Charon, J., Lecomte, G., Griboval, P., and Young, A. T.: 1968a, Astron. J. 73, 251 (Paper I).

Irvine, W. M., Simon, T., Menzel, D. H., Pikoos, C., and Young, A. T.: 1968b, Astron. J. 73, 807 (Paper II).

Murphy, R. E.: 1969, paper at Albany, N.Y., meeting of A.A.S.

O'Leary, B. T. and Rea, D. G.: 1968, Icarus 9, 405.

Wooley, R. v.d. R., Gottlieb, K., Heinz, W., and de Vaucouleurs, A.: 1955, Monthly Notices Roy. Astron. Soc. 115, 57. 